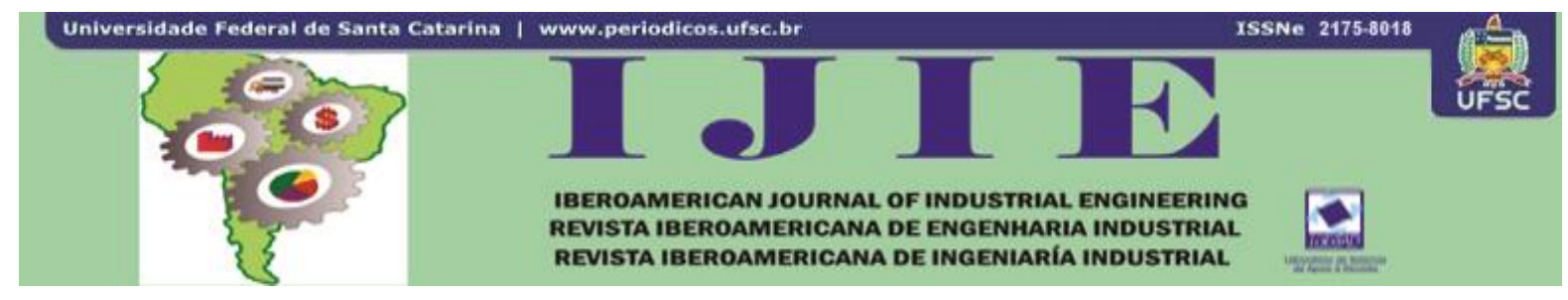

\title{
CONCEPTUAL MODEL FOR THE INTEGRATION OF PRONTO WITH ISO STANDARD 15926
}

\author{
Maria Soledad Sonzini ${ }^{1}$ \\ Marcela Vegetti ${ }^{2}$ \\ Horacio Pascual Leone ${ }^{3}$
}

\begin{abstract}
In this paper a conceptual model based on the ISO 15926 standard is proposed in order to extend and make explicit the representation of product variants given by PRONTO. This extension will allow introducing the product model in a collaborative environment where information systems are able to exchange information with other products systems. Interoperability of systems tends to increase the consistency of models, due to they may have several interpretations. Therefore, the proposal defines new elements and adapts these to the data model of the standard, for a complete concept mapping of PRONTO. Furthermore, changes in the product information are represented in time, through 4D approach.
\end{abstract}

Keywords: Interoperability. ISO 15926 Standard. Products Family. PRONTO. Semantic Web.

\section{INTRODUCTION}

The increase in demand for new products, the customer needs for a wider variety of items, the globalization of markets, the reduction of products lifecycle time, the need of increasing flexibility, the ability to response to the market changes, and the information technology improvement are the main challenges of companies that are involved in the product development process. The success of global manufacturing companies depends on the integration of their product development process and their manufacturing operations, which

\footnotetext{
${ }^{1}$ Systems Enginner and Ph.D. student in Engineering, Consejo Nacional de Investigaciones Científicas y Técnicas- Universidad Tecnológica Nacional, INGAR- Instituto de Desarrollo y Diseño (CONICET - UTN), (CP 3000), Avellaneda 3657, Santa Fe S3002GJC, Argentina. E-mail: ssonzini@santafe-conicet.gov.ar.

2 Doctor in Engineering, Consejo Nacional de Investigaciones Científicas y Técnicas- Universidad Tecnológica Nacional, INGAR- Instituto de Desarrollo y Diseño (CONICET - UTN), (CP 3000), Avellaneda 3657, Santa Fe S3002GJC, Argentina. E-mail: mvegetti@santafe-conicet.gov.ar.

3 Doctor in Engineering, Consejo Nacional de Investigaciones Científicas y Técnicas- Universidad Tecnológica Nacional, INGAR- Instituto de Desarrollo y Diseño (CONICET - UTN), (CP 3000), Avellaneda 3657, Santa Fe S3002GJC, Argentina. E-mail: hleone@santafe-conicet.gov.ar.
}

Iberoamerican Journal of Industrial Engineering, Florianópolis, SC, Brasil, v. 5, n. 10, p. 70-83, 2013. 
are geographically distributed. This integration can be achieved by means of a manufacturing collaborative environment (VALILAI; HOUSHMAND, 2013).

In manufacturing collaborative environments, the PLM (Product Lifecycle Management) systems are considered as information backbone of companies. PLM is the global term representing the management of product information throughout its life cycle (GOPSILL; MCAPLINE; HICKS, 2011). The various actors, consumers and/or producers of data from these systems require different views of the information because the type of information and the level of detail that is required by these actors are different. Thus, to achieve the implementation of these manufacturing environments, the corporate knowledge of different actors and several heterogeneous sources has to be unified (GRAUBE et al., 2011).

This unification requires a common model that enables the interoperability between different information systems involved during the product lifecycle management. This model, unlike the existing product models, hast to take into account the existence of multiple variants and versions for efficient product information management to enable rapid response to market changes.

In this sense, there are several proposals for representing and exchanging product data among different systems. The ISO 10303 (STEP - Standard for Exchange of Product model data) standard (STEP, 1991) is one of the most widely used. However, the application of this standard has encountered several difficulties (VALILAI; HOUSHMAND, 2013; LEE; EASTMAN; SACKS, 2007; BALL; DING; PATEL, 2008; GIELINGH, 2008). STEP represents product variants as different products with the consequent data duplication. On the other hand, it captures the information of products as a snapshot in time and lacks the ability to represent the products evolution over time.

These problems of STEP influenced the motivation for defining the ISO 15926 Part 2 standard. This standard describes a generic data model which allows the representation of product information at different abstraction levels. Moreover, it enables the representation of both the functional (abstract) and physical aspects of products.

Even though ISO 15926 has been defined for gas and oil production plants, due to its generality and extensibility capacity it has become a widely accepted standard in different industrial domains. It was designed keeping in mind a 4-dimensional vision of the data (4D approach). This approach allows representing product data evolution over time. However, this standard does not explicit how the product variants are specified. 
This paper proposes extend the ISO 15926 standard in order to make explicit the representation of product variants through the concepts proposed by PRONTO (PRoduct ONTOlogy) (VEGETTI; LEONE; HENNING, 2011). The mentioned ontology modifies the two-level product family concept (Family - Product) by the addition of a new level between them. This three level family concept (Family - VariantSet - Product) allows representing product information at different abstraction levels and it enables the definition of processes of information aggregation and disaggregation between them, This feature is important for production planning activities at different levels (strategic, tactical and operational).

This article is organized as follows. Section 2 introduces briefly the fundamental concepts of ISO 15926 standard and PRONTO ontology. The details of the proposed mapping are presented in Section 3 and its application into a case study is illustrated in Section 4. Finally, the conclusions and future work are drawn in Section 5.

\section{RELATED WORK}

Having in mind that the adoption of a neutral model contributes to the exchange of data in industrial plants, several standards focused on product data representation were proposed. One of these standards is ISO 15926 standard, which is widely accepted due to its generic data model. This generic data model is capable of representing product information in different domains. These specific product domain models are then implemented through repository-based web services and Semantic web technologies. Likewise, PRONTO is selected due to its capability of representing product variant in a consistently way. Both proposals are briefly introduced in this section.

PRONTO allows representing product data at different levels of abstraction. It defines two hierarchies, an abstraction $(\mathrm{AH})$ and a structural $(\mathrm{SH})$ ones. The first hierarchy allows the representation of non-structural product information at different abstraction levels as well as the definition of aggregation and disaggregation processes between these levels. The SH allows representing the information of products and the components required to manufacture them. PRONTO formalizes a knowledge representation that integrates the $\mathrm{SH}$ and $\mathrm{AH}$ hierarchies, emphasizing the treatment of structural information. As it is shown in Figure 1, the AH contains 3 levels:

- Family: Highest level of abstraction that represents a set of similar products that share one or more common structures.

Iberoamerican Journal of Industrial Engineering, Florianópolis, SC, Brasil, v. 5, n. 10, p. 70-83, 2013. 
- VariantSet: Intermediate level that represents a subset of family members, which share the same structure. These members may include changes on the structure of the family from which they belong.

- Product: Lower level of abstraction to represents individual items, which are members of a set of variants. They have a structure and represent products whit real existence.

Figure 1 - PRONTO main concepts

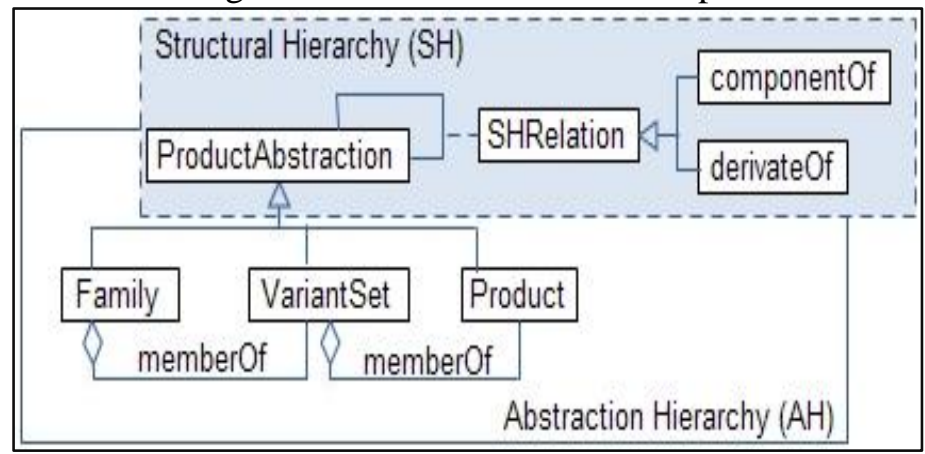

The memberOf association links these three levels and represents the membership relation between individuals that are defined at a lower level with an individual at the immediate upper level. The SH considers two types of structural relationship which are specialized as componentOf and derivateOf. The first one is used for structures that relate a product with the parts required to manufacture it. The second association links a product with the parts that are obtained from it by a disaggregation process. So, an entity may be related to other entities (components or derivatives) at the same level of abstraction. The SH relations allow the definition of different types of bills of materials (BOMs) for products that are made by assembling their component parts as well as by the disaggregation of non-atomic raw materials. In order to have an easier understanding of the proposal and narrow the scope of this article, it focuses only on the componentOf relationship.

In order to exemplify the two PRONTO hierarchies, a simple case study related with the assembly of DELL computer parts is considered. At the highest level of the AH the DELL Laptop Family is defined. This family has the following variant sets as members: DELL Inspiron 14z, Dell Inspiron 1545 and DELL Inspiron M5030. For space reasons, this example is focused only on the first variant set. DELL Inspiron $14 z$ has concrete products as members, like DELL Inspiron 14z 2100-SLV. 
As it is previously mentioned, the $\mathrm{SH}$ of a specific product specifies the components part required to manufacture it and it is defined at each level of the $\mathrm{AH}$. At the highest $\mathrm{AH}$ level, the SH of DELL Laptop Family involves the mentioned family and its component: Memory, Hard Drive and Processor families. At the middle level, the DELL Inspiron $14 z$ variant set is composed of DDR3, SATA HD and Dual Core variant sets, which are members of Memory, Hard Drive and Processor families respectively. In turn, the SH corresponding to DELL Inspiron $14 z 2100 S L V$ product is conform by 6 Gb DDR3 1600MHz, 500GB SATA HD, and Intel Core i3 $1.9 \mathrm{GHz}$, products which are members of the mentioned variant sets. More details about the mentioned example could be found in Section 4.

In turn, the ISO 15926 standard provides a neutral format to unify independent data formats for information systems interoperability in industries. It is organized into several parts, many of which are still under evaluation. This work pays special attention to the data model included in ISO 15926 Part 2. This data model will be extended in order to represent the $\mathrm{AH}$ and $\mathrm{SH}$ hierarchies aforementioned. ISO 15926 Part 4 creates a reference data library (RDL-Reference Data Library) from Part 2 data model. This library organizes all the elements defined in tabular form with a unique identification number, a name in English or Russian, a brief description, the entity type from which the element is instance of, and some additional data (ISO/TS 15926-4, 2012).

Table 1 - Main entities types of the data model of ISO 15926 standard

\begin{tabular}{|l|l|}
\hline $\begin{array}{l}\text { Thing: It is anything that is or may be thought about } \\
\text { or perceived, including material and non-material } \\
\text { objects, ideas, and actions. }\end{array}$ & $\begin{array}{l}\text { functional_physical_object: it is a physical_object } \\
\text { that has functional, rather than material, continuity as } \\
\text { its basis for identity. }\end{array}$ \\
\hline $\begin{array}{l}\text { class_of_class_of_individual: It is a class_of_class } \\
\text { whose members are instance of class_of_individual. }\end{array}$ & $\begin{array}{l}\text { physical_object: It is a possible_individual that is a } \\
\text { distribution of matter, energy or both. }\end{array}$ \\
\hline $\begin{array}{l}\text { class_of_class_of_composition: it indicates that its } \\
\text { members are instance of class_of_composition_of__ } \\
\text { individual. }\end{array}$ & $\begin{array}{l}\text { thaterialized_physical_object: it is a physical_object } \\
\text { identify. }\end{array}$ \\
\hline $\begin{array}{l}\text { Beginning / ending: It is a temporal_bounding that } \\
\text { marks the temporal start/end of a possible_individual }\end{array}$ & $\begin{array}{l}\text { class_of_relationship: it is a class_of_abstract_object } \\
\text { whose members are members of relationship. }\end{array}$ \\
\hline $\begin{array}{l}\text { class_of_composition_of_individual: It is a } \\
\text { class_of_relationship whose members are instance of } \\
\text { composition_of_individual. }\end{array}$ & $\begin{array}{l}\text { event: it is a possible_individual that indicate a } \\
\text { temporal boundary between one or two possible } \\
\text { individual. }\end{array}$ \\
\hline $\begin{array}{l}\text { possible_individual: It is a thing that exists in space } \\
\text { and time. }\end{array}$ & $\begin{array}{l}\text { temporal_bounding: It indicates that an } \text { event is a } \\
\text { temporal bound. }\end{array}$ \\
\hline $\begin{array}{l}\text { composition_of_individual: it indicates that a } \\
\text { possible_individual is part of possible_individual. }\end{array}$ & $\begin{array}{l}\text { class_of_individual: It is a class whose members are } \\
\text { instance of possible_individual }\end{array}$ \\
\hline
\end{tabular}

There are diverse RDLs that cover different areas of definition. Due to their size have been increased over time causing difficulties to read the tables, a set of templates have been Iberoamerican Journal of Industrial Engineering, Florianópolis, SC, Brasil, v. 5, n. 10, p. 70-83, 2013. 
developed in Part 7 of the standard. These templates have the aim of creating a common neutral framework for exchange information among systems. Thus, using these templates, information from different sources is mapped to this common structure to reach interoperability.

The use of templates ensures that the entities and relationships used in the RDL have a precise meaning, which facilitates the reusability and interoperability among information systems (JOHAN; KLÜWER; SKJÆVELAND, 2008). Templates are required for the implementation of Part 8 of the standard, which allows mapping the content of templates into OWL (Ontology Web Language) statements. Currently there are about 240 templates defined and categorized (LIST OF TEMPLATES, 2013), many of which are being evaluated. Some basics concepts were shown and briefly described in Table 1. These concepts are also presented in Figure 2 and they are mentioned in the following sections.

Figure 2 - Representation of abstraction levels whit ISO 15926 Parte 2

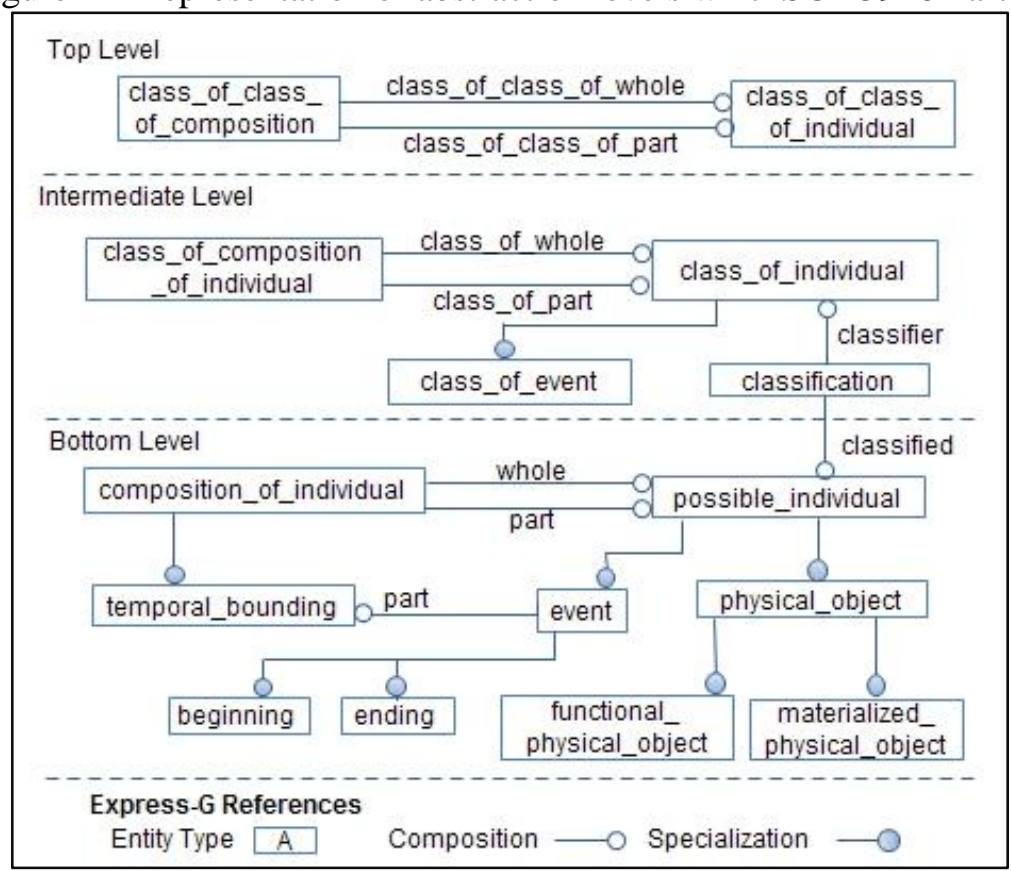

The proposed concepts in the ISO 15926 data model allow defining 3 information abstraction levels: Top, Bottom, and Intermediate. The highest level ("Top Level"), which is represented by class_of_class_of_individuals entity type, relates to other entities in the same level through the composition relationship called class_of_class_of_composition. In this relation, an entity plays the role of compound object or container (class_of_class_of_whole) and the other entity plays the role of a component or part (class_of_class_of_part). 
To represent an intermediate level of abstraction ("Intermediate Level") the data model provides the class_of_individual entity type. This type relates to others entities at the same level using the class_of_composition_of_individual composition relationship in which the participants play the role of class_of_part and class_of_whole respectively. The lowest abstraction level ("Bottom Level") is represented by the possible_individual entity type which can represents both physical objects (physical_object) as well as events (event). The composition association at this level is defined by composition_of_individual entity type. The entity types that participate in this relation play the roles of whole and part, respectively.

As it is shown in Figure 2, the classification entity type allows connecting a possible_individual at the lowest level with its class (class_of_individual) at the intermediate level. In this relationship the mentioned entities types play the roles of classified and classifier, respectively.

In addition, the standard introduces the 4D spatial-temporal approach for the definition of physical objects. According to this approach, each physical_object has two parts, one temporal and one spatial. For the representation of these parts, the approach introduces two entity types: functional_physical_object and materialized_physical_object. The first entity type expresses the function that a physical object has to execute. The second one indicates that the physical object is playing some specific role in a given period. The length of this period is defined by the occurrence of events that indicate the beginning ("beginning") or the end ("ending") of the object on the temporal path.

\section{MAPPING PRONTO TO ISO 15926 STANDARD}

The three levels of product definition given by PRONTO were represented using different levels of abstraction available in the standard data model. The type of class_of_class_of_individual entity, which is defined at the highest level of abstraction in the ISO 15926 standard, represents the concept of family as is specified in the ontology. Similarly, class_of_individual and physical_object represents Variant Set and Product concepts, respectively. The composition relations between entities of the same level, which are called class_of_class_of_composition, class_of_composition_of_individual, and composition_of_individual allow representing the SH componentOf association in each level of PRONTO abstraction hierarchy.

Iberoamerican Journal of Industrial Engineering, Florianópolis, SC, Brasil, v. 5, n. 10, p. 70-83, 2013. 
For the representation of the AH member $O f$ relationship the proposal suggests to use the classification entity type that links the product level playing the role of classified, with Variant Set level having the classifier role. The standard does not include an entity type that links the upper level, which represents the family, with the intermediate level representing the variant set concept. This is because generally, in other proposals for implementing the product family concept only two levels (the family and its members) are considers, unlike PRONTO which establishes 3 abstraction levels. In order to represent the memberOf relationship between the two upper levels, the definition of a new entity type called class_of_classification is proposed.

The entity type class_of_classification is subclass of class_of_relationship and superclass of classification (see Figure 3). Both class_of_relationship and classification are basic entities types of the ISO 15926-2 data model (see Table 1). The entity type class_of_classification sets that class_of_individual, with class_of_classified role, is a member of class_of_class_of_individual having the class_of_classifier role. Thus, the VariantSet level is linked to the Family one reaching the binding of the 3 levels proposed by PRONTO. Therefore, a complete representation of PRONTO AH and SH hierarchies is achieved. Figure 3 shows the proposed new elements and the corresponding mapping between PRONTO model and the data model of Part 2 of the standard.

Figure 3 - Mapping PRONTO to ISO 15926

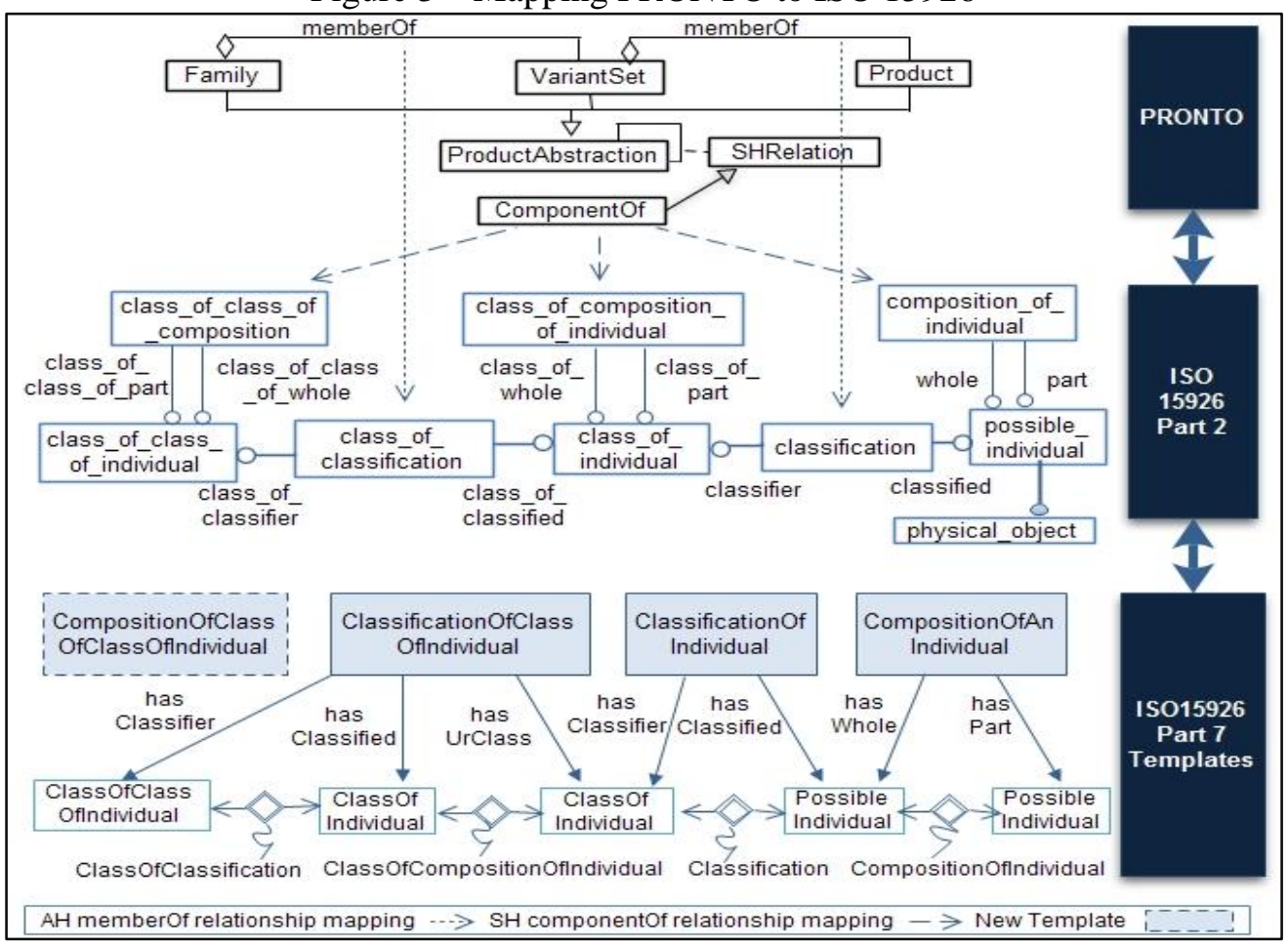

Iberoamerican Journal of Industrial Engineering, Florianópolis, SC, Brasil, v. 5, n. 10, p. 70-83, 2013. 
To simplify the understanding of the mapped data model three templates, which are defined in Part 7 of the standard, are considered. These templates allow representing the product information as shown in Figure 3. Each template has a name, a specification in firstorder logic and the URI (Unified Resource Identifier) of each element that composes it. The template CompositionOfAnIndividual allows representing componet $O f$ relationship at Product level. In turn, the template ClassificationOf-ClassOfIndividual is proposed to represent the same relation at the Variant Set level and memberOf relationship between this intermediate level and the Family level.

ClassificationOfIndividual template enables the representation of memberOf association between the lowest and intermediate levels. Finally, to complete the representation of the relationships of both PRONTO hierarchies, the definition of a new template is proposed with the name CompositionOfClassOfClassOfIndividual to specify the componentOf relation at the highest level. The definition of this new template has been necessary to maintain the representation of the three different levels. In Table 2 the relevant aspects of the specification of the template are shown using the format published in the web site POSC Caesar Association (BATRES et al., 2007) for templates specification.

Table 2 - CompositionOfClassOfClassOfIndividual template specification

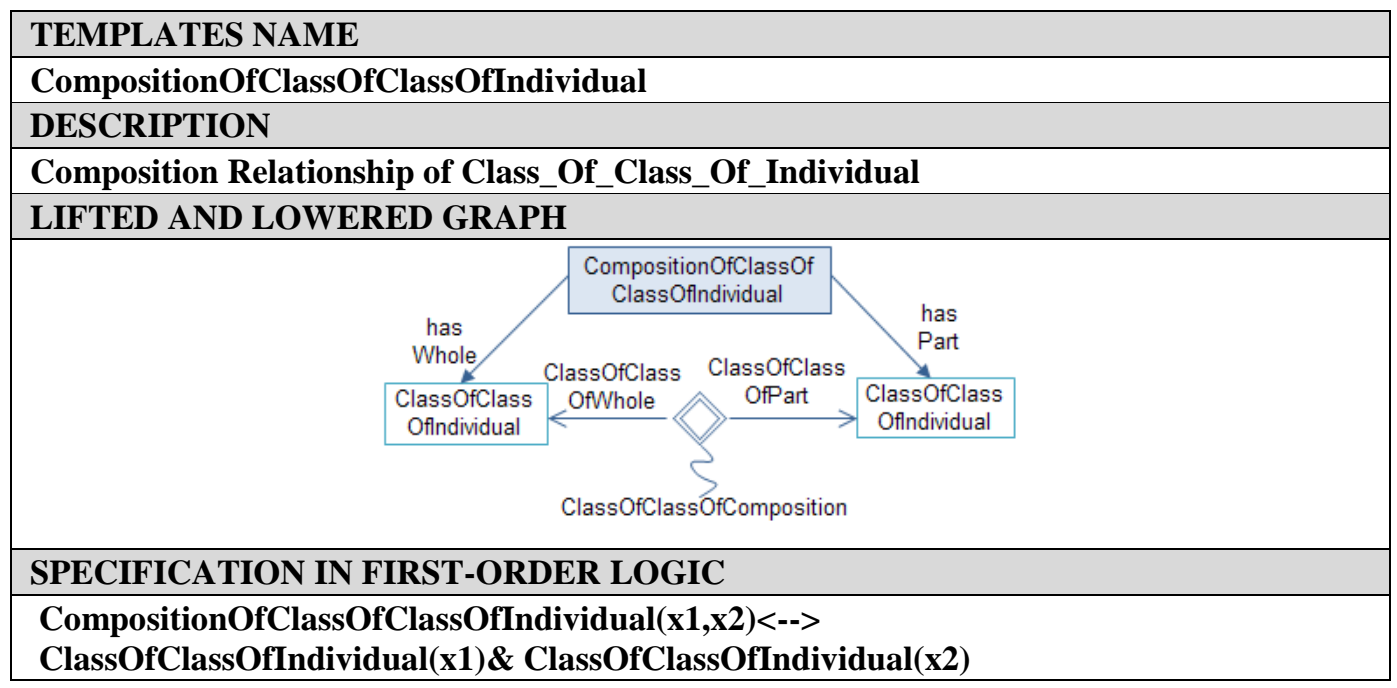

At Product level, objects can have functional continuity rather than material one, and their components can be replaceable parts (materialized_physical_object) without affecting the function of the whole object (functional_physical_object). This situation may be represented by the 4D approach proposed by ISO 15926. The 4D approach applies only at the 
lowest level of definition of information products, because of their physical existence. However, in section 4 the mentioned approach will be adapted to be used at the higher abstract levels. The modification proposed in the mentioned section suggests the use of overlapping layers reflecting product versions at different levels. The representation of versions may affect the consistency of PRONTO model. Therefore, it is necessary the definition of a mechanism for version traceability that manages versions and product history. However, for space limitation this aspect is not considered in the present work.

\section{CASE STUDY}

In this section, is describing the product representation of an organization responsible for the assembly of computer parts. Figure 4 shows part of the family DELL Laptop abstract hierarchy. This hierarchy binds through the member $O f$ relation the mentioned family with its DELL Inspiron 14z Variants Set (CV). DELL Inspiron 14z Variants Set has a DELL Inspiron $14 \mathrm{z} 2100 \mathrm{SLV}$ Product as its member. The componentOf relationship states that the DELL Laptop family structure includes Memory, Hard Drive and Processor families. Similarly, the SH of DELL Inspiron 14z CV contains others variants sets as: DDR3, SATA HD and Dual Core.

Each CV is linked with a family at top level and with at least one product at lower level, conforming the AH (show in Figure 4 by the dotted arrows). The hierarchy abstraction relationships with the lower level are not shown to maintain the clarity of Figure 4, but these relationships are similar to the top level explained. The Level Product (see Fig. 4) shows the SH of DELL Inspiron 14z 2100sLV product is shown.

Each element mentioned in the previous paragraphs are instances of entity types of the data model proposed in the ISO 15926 (ISO 15926, 2013; TEMPLATE SPECIFICATION, 2013) Part 2 standard. Thus, each family is instance of the class_of_class_of_individual entity type and can have a structure composed by other families or not (i.e. Dell Laptop).

In the first case, a composition structure has the class_of_class_of_composition relationship to link the DELL Laptop family, which is instance of class_of_class_of_whole, with its parts that are instances of class_of_class_of_part. Each family has a CV associated by memberOf relationship, which is an instance of the class_of_classification entity type proposed in the previous section. In this relationship, the family DELL Laptop plays the role of class_of_classifier and DELL Inspiron $14 z \mathrm{CV}$ plays the role of class_of_classified. 
Figure 4 - Data Model of Case Study

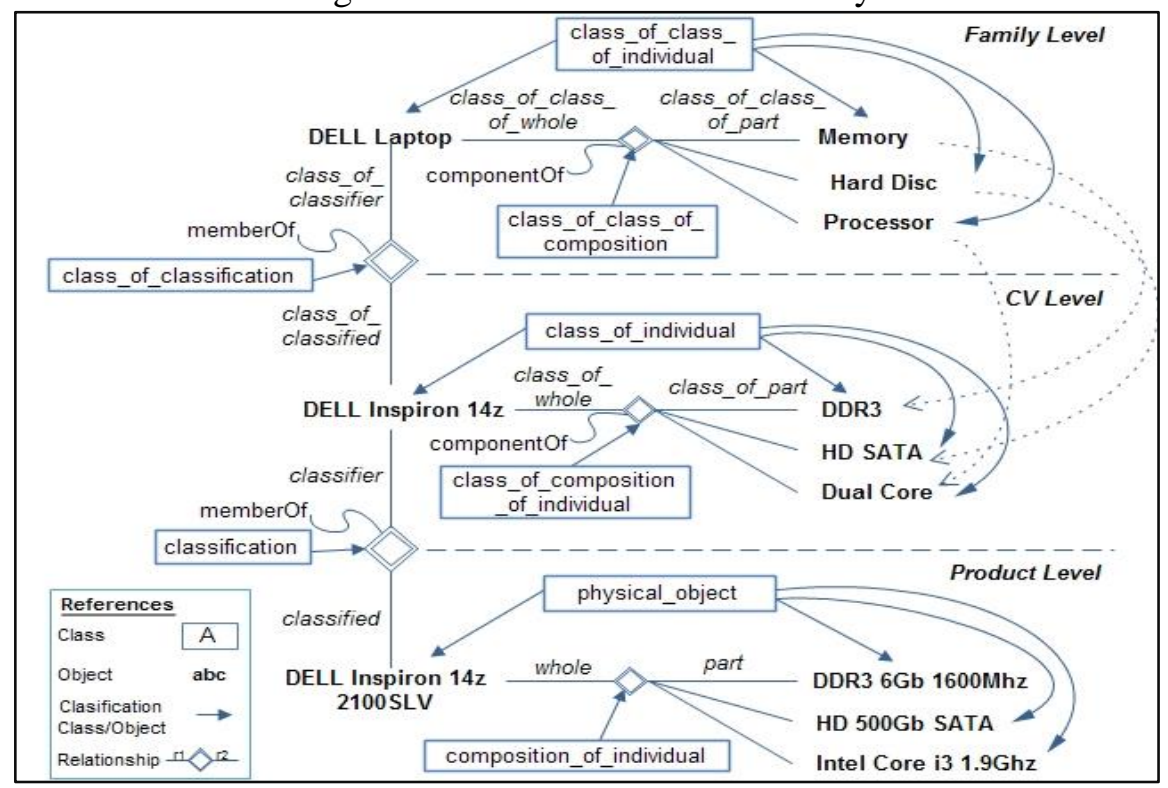

At the CV level, each CV is represented as instances of class_of_individual and the composition relationships between entities at this level as instances of class_of_composition_of_individual. In this relationship the Dell Inspiron $14 z$ plays the role of class_of_whole and the other sets shown (DDR3, SATA HD Driver and Core) play the role of class_of_part.

At the Product level, the products are represented as instances of physical_object, a possible_individual subclass. Each product is relates with a $\mathrm{CV}$ through the instantiation of the classification entity type, where the CV plays the role of the classifier and the product of classified. The SH relationship at Product level corresponds to an instance of the composition_of_individual entity type.

4D approach representation considers the occurrence of an event indicating a change in the structure information of a product. For example, the event could be an inconvenience with the import process of Processor component, which implies the need to replace it with another compatible model and identify the product version to facilitate the information traceability.

In Figure 5, the replacement of the $1.9 \mathrm{Ghz}$ Intel Core i3 product by the $2.4 \mathrm{Ghz}$ Intel Core i3 product is shown. As mentioned above, the 4D approach applies to products with physical existence, represented at the Product level. However, in Figure 5 the three abstraction levels are represented by the superposition of layers incorporating an additional axis on the traditional graphic representation of the standard. 
Figure 5-4D approach representation including abstraction levels

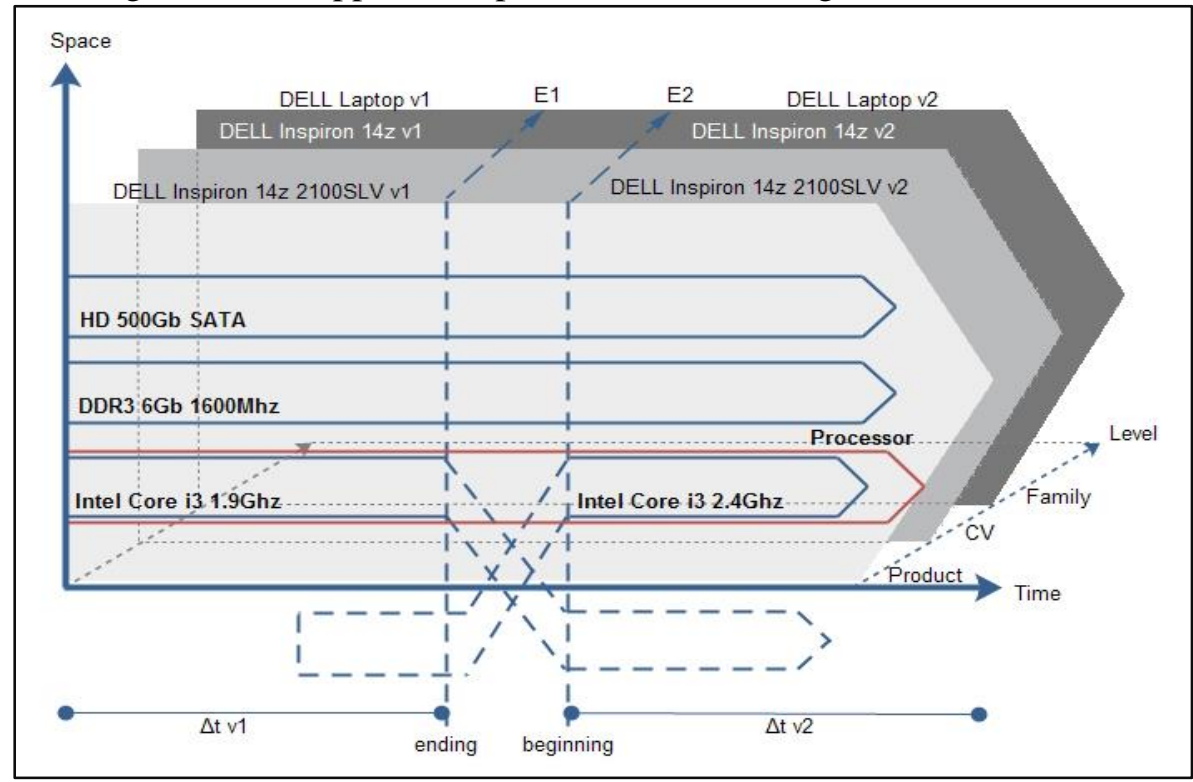

During the change of products in Figure 5, the functional continuity Processor (functional_physical_object) is not affected, because both products develop the same function and are fully replaceable. The event E1, besides indicating the end (ending) of $1.9 \mathrm{Ghz}$ Intel Core i3 product, shows that the product information has undergone a change ending the version 1, where $\Delta \mathrm{t} v 1$ shows its trajectory over time. The event E2 indicates the starting point (beginning) of the materializing (materialized_physical_object) of the processor function with a new product version, Intel Core i3 $2.4 \mathrm{Ghz}$.

Dotted areas in Figure 5 represent the physical continuity of the product. This implies that, despite replacing the $1.9 \mathrm{Ghz}$ Intel Core i3 product which materializes Processor function in a product of Dell Laptop family, this object (Intel Core i3 1.9Ghz) can materialize the function in another products family dissimilar to this case study. This analysis can be applied on the CV level and Family level. However, the replacement of any component affects the lower-level level. This means, if the HP Dual Core CV component is replaced by another HP Quad Core CV, the Intel Core i3 1.9Ghz component, which belongs to the product level (the lowest level), will be affected due to the consistency in the AH relationship of PRONTO.

\section{CONCLUSIONS AND FUTURE WORK}

These days, industries recognize the importance of the integration of different information systems as a key to be inserted and compete in the global market with increasing consumer demands. Integration requires a neutral data model capable of supporting different

Iberoamerican Journal of Industrial Engineering, Florianópolis, SC, Brasil, v. 5, n. 10, p. 70-83, 2013. 
data formats, specific to each industry, to achieve interoperability of their information systems. Therefore this proposal presents a mapping between the concepts of standard ISO 15926 and ontology PRONTO. This strategy seeks to integrate, through the ISO 15926 standard, the ontology PRONTO with other ontologies to facilitate information systems interoperability. The standard provides a user-oriented language to represent, assess and improve operations, but it lack of tools to represents domain models.

Moreover, the ISO 15926 standard, unlike STEP, has the advantage of having a low coupling extensible information model. The data model described in Part 2 is quite robust and allows us to represent most of the product information introduced by the ontology PRONTO. To complete this representation, it was necessary to define a new entity type and a template for the complete mapping of the two proposals (PRONTO and standard ISO 15926), because the levels of abstraction of the ontology were not fully represented in the standard.

Currently, Part 7 of ISO 15926 standard is under development and many of the proposed templates are still being evaluated. The advantage of having defined templates (in Part 8 of the standard) is that they can be transformed into statements in OWL language, with the aim of carrying out an implementation based on Semantic Web technologies.

As future work, a model to represent versions of the product information, maintaining consistency with the different levels of definition will be developed. In addition, we will work on the specification of a single template applied to a particular domain, to simplify the mapping between PRONTO and ISO15926 standard.

Acknowledgements. This work was financed jointly by CONICET (PIP 11220110100906), the National Agency of Scientific and Technological Promotion (PICT 2315), the National Technological University (PID 25-O156 and PID 25-O144), and the National University of La Rioja. The support provided by these institutions is gratefully acknowledged.

\section{REFERENCES}

BALL, A.; DING, L.; PATEL, M. An approach to accessing product data across system and software revisions. Advanced Engineering Informatics, v. 22, p. 222-235, 2008. 
BATRES, R.; WEST, M. LEAL, D.; PRICE, D.; MASAKI, K.; SHIMADA, Y.; FUCHINO, T.; NAKA Y. An upper ontology based on ISO 15926. Computers \& Chemical Engineering, v. 31, n. 5-6, p. 519-534, 2007.

DATA MODEL ISO 15926. Part 2. Retrieved from: <http://www.infowebml.ws>

GIELINGH, W. An assessment of the current state of product data technologies. ComputerAided Design, v. 40, p. 750-759, 2008

GOPSILL, J.; MCAPLINE, H.; HICKS, B. Trends in Technology and their Possible Implications on PLM: Looking Towards 2020. Proceedings..., of International Conference on Product Lifecycle Management, 2011.

GRAUBE, M.; PFEFFER, J.; ZIEGLER, J.; URBAS, L. Linked Data as integrating technology for industrial data. Proceedings..., International Conference on Network-Base Information Systems, 2011.

ISO 15926-4. Under the ISO/TS. 2012. Retrieved from: <http://www.caesarsystems.co.uk/iso/ts/15926/-4/tech/reference-data-library/v-0/> JOHAN W.; KLÜWER, M.G.; SKJÆVELAND, M.V-S. ISO 15926 templates and the Semantic Web. 2008. Retrieved from: <http://www.w3.org/2008/11/ogwspapers/kluwer.pdf>

LEE G.; EASTMAN, C.; SACKS, R. Eliciting information for product modeling using process modeling. Data \& Knowledge Engineering, v. 62, p. 292-307, 2007.

LIST OF TEMPLATES. 2013. Retrieved from: <http://www.infowebmlws/TS/TSdetails/index.htm>

STEP. Product Data Representation and Exchange-Part 44. Product Structure Configuration. Standard ISO 10303, 1991.

TEMPLATES SPECIFICATION. Retrieved from: $\langle$ http://15926.org/15926_template_specs.php $>$.

VALILAI, O.F.; HOUSHMAND, M. A collaborative and integrated platform to support distributed manufacturing system using a service-oriented approach based on cloud computing paradigm. Robotics and Computer-Integrated Manufacturing, v. 29, p.110$127,2013$.

VEGETTI, M.; LEONE, H.; HENNING, G. PRONTO: An ontology for comprehensive and consistent representation of product information. Engineering Applications of Artificial Intelligence, v. 24, n. 8, p. 1305-1327, 2011.

Originais recebidos em: 26/10/2013

Aceito para publicação em: 15/04/2014

Iberoamerican Journal of Industrial Engineering, Florianópolis, SC, Brasil, v. 5, n. 10, p. 70-83, 2013. 